\title{
Capacidad de depredación de Chrysoperla carnea sobre Nysius sp. Plaga de la quinua, a nivel de laboratorio en la irrigación Majes - 2017
}

Depredation capacity of Chrysoperla carnea on Nysius sp. pest of Chenopodium quinoa, laboratory level in the irrigation Majes - Arequipa 2017

Dina Beatriz Mamani Gutierrez, Celia Martha Pinto Manrique

Autoridad Autónoma de Majes (Autodema), Irrigación Majes, Distrito de Caylloma, Provincia y departamento de Arequipa.

\section{INFORMACIÓN}

\section{Historia del Artículo \\ Recepción: 26/05/2019 \\ Revisión: $15 / 07 / 2019$}

Aceptación: 18/08/2019

\section{Palabras Clave}

Capacidad de depredación, Chrysoperla carnea, ninfas de Nysius $s p$., adultos de Nysius sp.

\section{Key Words}

Predation capacity, Chrysoperla carnea, nymphs of Nysius sp., Adults of Nysius sp.

\section{DOI}

https://doi.org/10.35286/veritas. v20i2.247

\begin{abstract}
RESUMEN
El presente trabajo de investigación se desarrolló en el laboratorio de AUTODEMA en la Irrigación de Majes en el año 2017, a fin de evaluar la capacidad de depredación de Chrysoperla carnea (Larva III) como controlador biológicos de Nysuis sp. en sus estadios ninfales y adulto; en el cultivo de quinua en la Irrigación de Majes, siendo el principal objetivo de la investigación. A fin de realizar esta experimentación se obtuvieron especies Chrysoperla carnea del laboratorio de control biológico de Servicio Nacional de Sanidad Agraria SENASA - Lima, realizando una crianza masal en sus diferentes estadíos y para la obtención de individuos de Nysius sp., se hizo recolección de insectos de las áreas de quinua de la irrigación Majes; los cuales fueron criados en condiciones de laboratorio. Para evaluar la capacidad de depredación de la especie antes mencionada se desarrolló un estudio donde se tuvieron dos tratamiento T1 (ninfas de Nysius $s p$.) y T2 (adultos de Nysius sp.), ambos tratamientos tuvieron 5 repeticiones y la evaluación se realizó durante 5 días consecutivos hasta que el total de larvas de crisopas pasaron a su estado de pupa, con lo que concluyeron las evaluaciones. Los resultados obtenidos indican que Chrysoperla carnea en su estadio larval III es el más voraz sobre ninfas que adultos de Nysius $s p$.; donde una larva durante todo su estadio larval III depreda en promedio 25 ninfas y 9 adultos.
\end{abstract}

\begin{abstract}
The present research work was developed in the AUTODEMA laboratory in Majes Irrigation in 2017, in order to evaluate the predation capacity of Chrysoperla carnea (Larva III) as biological controller of Nysuis sp. in its nymphal and adult stages; in the cultivation of quinoa in Majes Irrigation, being the main objective of the investigation. In order to carry out this experimentation, they obtained Chrysoperla carnea species from the Biological Control Laboratory of the National Agrarian Health Service SENASA - Lima, carrying out a mass breeding in their different stages and obtaining individuals from Nysius sp., collecting insects of the quinoa areas of the Majes Irrigation; which were reared in laboratory conditions. To evaluate the predation capacity of the species mentioned above, a study was carried out where two T1 treatments (Nysius sp. Nymphs) and T2 (Nysius sp. Adults) were taken. Both treatments had 5 repetitions and the evaluation was carried out for 5 days. consecutive until the total lacewings larvae went to pupal status, which concluded the evaluations. The obtained results indicate that Chrysoperla carnea in its larval stage III is the most voracious on nymphs that adutos of Nysius sp .; where a larva throughout its larval stage III depredates on average 25 nymphs and 9 adults.
\end{abstract}

\section{INTRODUCCIÓN}

El control biológico que es la base del manejo integrado de plagas (MIP); contribuye en la conservación de los ecosistemas agrícolas, permitiendo a las empresas exportadoras reducir costos, cumplir con las medidas fitosanitarias internacionales y apoyar la preservación del ambiente y la salud.

Chrysoperla carnea es un depredador generalista y voraz en sistemas agrícolas (Tauber et al., 2000), presenta amplio intervalo de presas (McEwen et al., 2001), es efectivo como agente de control biológico.

Correspondencia:

Dina Beatriz Mamani Gutierrez

dmamanig@ucsm.edu.pe
El estadio larval es el que realiza el control, siendo las larvas de tercer estadio las que ejecutan la mayor parte del trabajo, alimentándose de insectos de cuerpo blando tales como áfidos, moscas blancas y trips entre otros. (Loera et al 2001)

\section{MATERIALES Y MÉTODOS}

\section{Materiales}

Material biológico (adultos y ninfas de Nysius sp., larvas de tercer estadio de Chrysoperla carnea y huevos de Sitotroga cerealella), glomérulos de quinua limpios, tapers de plástico $125 \mathrm{ml}$. y 10 1. de capacidad), tela organza, pegamento, vasos pequeños de plástico, algodón, papel aluminio, aspirador de insectos, estereoscopio, plumón indeleble y cartillas de evaluación. 


\section{Metodología}

\section{Obtención Chrysoperla carnea larva III}

Las larvas de tercer estadio se obtuvieron a partir de huevecillos proporcionados por el laboratorio de control biológico de Servicio Nacional de Sanidad Agraria SENASA, Lima. Estos huevecillos fueron separados en vasos de plástico. Luego de su emergencia, las larvas fueron alimentadas con huevos de Sitotroga cerealella hasta obtener larva III.

\section{Crianza de Nysius $s p$.}

Se recolectó especímenes (ninfas y adultos) de campos de quinua y se instalaron en tapers grandes (10 1. de cap.) Los cuales fueron condicionados con una abertura cubierta con organza (caja de crianza); donde se colocó el material biológico recolectado y se les alimentó con glomérulos de quinua por un periodo de 4 semanas. Con lo que las poblaciones incrementaron y se utilizaron las ninfas $y$ adultos para iniciar los ensayos.

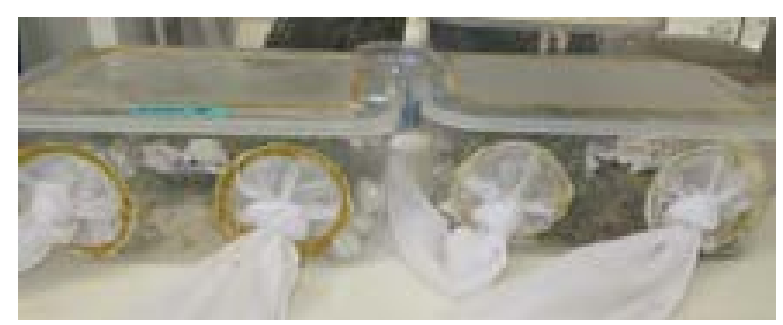

Fig. 1: Cajas de crianza Nysius sp.

\section{Instalación del ensayo}

El área experimental fue una caja de crianza (pequeña 125 ml cap.) para cada tratamiento, donde se colocó diariamente un glomérulo de quinua, sin infestación de chinches, para mantener la turgencia del glomérulo, el pedicelo fue envuelto con un pedazo de algodón humedecido en agua y cubierto con un trozo de papel aluminio. Esté glomérulo fue la fuente de alimento para los chinches (Nysius $s p$ ) que fueron ofrecidos como presas a los depredadores Chrysoperla carnea.

En cada área experimental se depositaron 30 individuos de Nysius $s p$, tanto ninfas (de diferentes estadios) y adultos. En cada caja de crianza se introdujo un espécimen (larva III) de la especie depredadora y se mantuvo durante 24 horas. El bioensayo para cada larva contó con 5 repeticiones.

Una vez que se completaron las 24 horas se cambió el glomérulo y se colocó nuevamente 30 especímenes con el depredador puesto inicialmente, esto se repitió hasta que el depredador paso a su estado pupal con lo que concluyó en ensayo. Cada 24 horas se realizaron las evaluaciones donde se realizó el conteo de especímenes consumidos. Los chinches consumidos diariamente se contabilizaban bajo el estereoscopio.

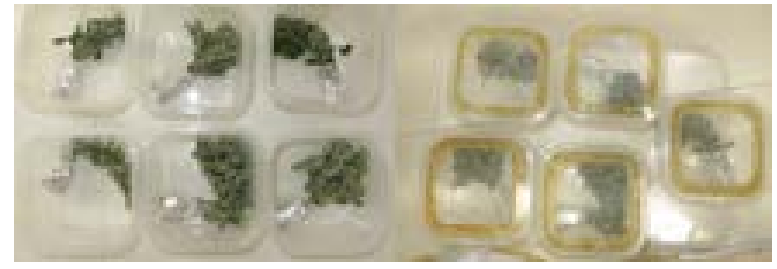

Fig. 2: Preparación e instalación de las áreas experimentales

\section{RESULTADOS}

Para realizar las evaluaciones de la capacidad de depredación de Chrysoperla carnea sobre ninfas y adultos de Nysius sp. se consideraron los insectos muertos que presentan algún daño en la zona del cuerpo; siendo esto observado a través del estereoscopio.

Tabla 1: Número especímenes depredados por día

\begin{tabular}{ccc}
\hline \multirow{2}{*}{ DÍA } & \multicolumn{2}{c}{ NÚMERO DE INDIVIDUOS PREDATADOS } \\
\cline { 2 - 3 } & NINFAS & ADULTOS \\
\hline 1 & 39 & 0 \\
2 & 48 & 17 \\
2 & 25 & 24 \\
4 & 10 & 3 \\
5 & 2 & 2 \\
\hline TOTAL & 124 & 46 \\
\hline PROMEDIO DE & & \\
INDIVIDUOS & & 9 \\
DEPREDADOS EN & 25 & \\
UN DIA POR UN & & \\
ESPÉCIMEN DE & & \\
Chrysoperla & & \\
\hline
\end{tabular}

Se puede observar que las ninfas son presas más atractivas en comparación a los adultos, también se observa que en los tres primeros días del estadio larval III, para el caso de los especímenes alimentados con ninfas existe un mayor consumo de individuos, los dos últimos dos días el consumo baja considerablemente pudiendo deberse a que la larva está empezando a bajar sus actividades por estar próximo el estadio de pupa.

En estado adulto se observa que no hay un buen porcentaje de depredación por día (9\%) pudiendo ser atribuido al cuerpo de los adultos son más duros y por consiguiente no tan apetecible para el depredador.

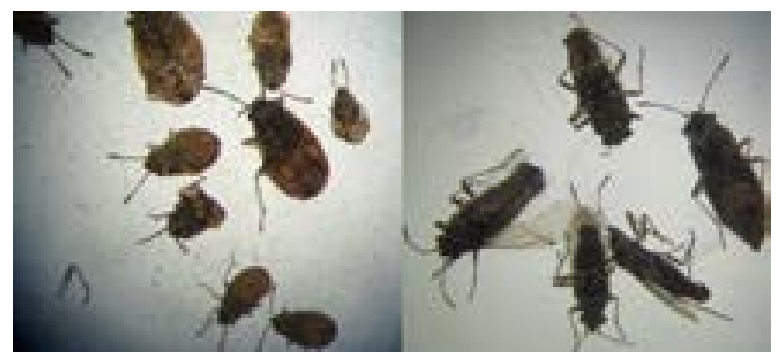

Fig. 3: Ninfas y adutos de Nysius sp. depredados por Chrysoperla carnea 


\section{CONCLUSIONES}

- Se observa que existe una mayor preferencia de alimentación por los estadios ninfales que los adultos.

- En sus primeros tres día del estado larval 3 la Chrysoperla carnea; presenta un mayor número de presas devoradas.

- Las larvas III de Chrysoperla carne no tiene facilidad para predatar adultos, lo cual se considera que ocurre debido a la cuticular endurecida de estos.

\section{AGRADECIMIENTO}

A la empresa AGROUNIMEX S.A. quienes a través del proyecto "Tecnología para el control eficiente del complejo de Chinches en cultivo de quinua (Chenopodium quinoa) en la Irrigación Majes", según convenio PITEI-2-P-111-027-14, financió el desarrollo de este trabajo de investigación.

\section{REFERENCIAS BIBLIOGRAFICAS}

1. Loera et al. (2001) disponible en: http://www.scielo. org.co/pdf/rcia/v33n2/v33n2a04.pdf

2. McEwen, P. K., T. R. R. New, and A. Whittington. 2001. Disponible en: http:// www.scielo.br/scielo.php? script $=\mathrm{sci}_{-}$ arttext\&pid=S1808-16572015000100307

3. Tauber, M. J., C. A. Tauber, K. M. Daane, and K. S. Hagen. 2000. Commercialization of predators: recent lessons from green lacewings. Disponoble en : http:// zsp.com.pk/pdf43/1049-1054\%20(4)\%20PJZ-53310\%2018-10-2010.pdf 